\title{
ANALYSIS OF BASIS WEIGHT UNIFORMITY INDEXES FOR THE EVALUATION OF FIBER INJECTION MOLDED NONWOVEN PREFORMS
}

\author{
Patrick Moll*, Shaofan Wang, Sven Coutandin, Jürgen Fleischer \\ Karlsruhe Institute of Technology, wbk Institute of Production Science, Kaiserstraße 12, 76131 Karlsruhe, Germany \\ ${ }^{*}$ Corresponding author. E-mail: patrick.moll@kit.edu
}

\begin{abstract}
:
Fiber injection molding is an innovative approach for the manufacturing of nonwoven preforms but products currently lack a homogeneous fiber distribution. Based on a mold-integrated monitoring system, the uniformity of the manufactured preforms will be investigated. As no universally accepted definition or method for measuring uniformity is accepted yet, this article aims to find a suitable uniformity index for evaluating fiber injection molded nonwovens. Based on a literature review, different methods are implemented and used to analyze simulated images with given distribution properties, as well as images of real nonwovens. This study showed that quadrant-based methods are suitable for evaluating the basis weight uniformity. It has been found that the indexes are influenced by the number of quadrants. Changes in sample size do not affect the indexes when keeping the quadrant number constant. The quadrants-based calculation of the coefficient of variation showed the best suitability as it shows good robustness and steady index for varying degrees of fiber distribution.
\end{abstract}

\section{Keywords:}

Fiber injection molding; nonwovens; glass fiber; preform; basis weight uniformity; image processing

\section{Introduction}

Due to the scarcity of resources and energy, stricter legislation regarding fuel consumption and emissions in the transport sector, and the growing environmental awareness among the population, the importance of system-efficient lightweight construction has increased significantly in recent years. Fiber-reinforced plastics (FRPs) have a high potential due to their density-specific very good stiffness and strength [1]. Currently, the use of FRPs is limited due to the high material and manufacturing costs in mass production [2]. Nonwovenbased composites represent a cost-effective alternative in semistructural applications such as claddings [3]. For the manufacturing of nonwoven preforms, sequential processes are currently used, where the contour is cut from nonwovens and mats, which are available as two-dimensional (2D) semifinished products in the form of rolled goods, and consecutively formed [4]. This goes hand in hand with corresponding waste, which leads to higher material costs.

A promising approach for the direct production of threedimensional (3D) nonwoven preforms without the intermediate step of 2D semifinished products is the fiber injection molding (FIM) process [1]. The process allows the production of 3D long-fiber semifinished products without cutting waste by blowing fibers into a closed mold by means of an air stream [5]. This process has the potential to locally adjust the density of the fiber structure. One impediment to the use of FIM as a preforming process is the varying mass weights of the fiber structure achievable to date, which leads to problems during subsequent infiltration [6]. This inconsistency of FIM nonwovens can be attributed to the injection process step where the fibers are deposited in the mold [7]. With the goal to better understand the injection process and the web formation within the mold, a process monitoring system has been developed [8]. This monitoring system consists of a camera integrated in the injection mold which records the fiber injection. The images of this camera will be used to measure the basis weight uniformity of the nonwoven online during the manufacturing process.

Despite several studies dealing with the uniformity analysis of nonwovens, no universally accepted definition or method is accepted yet [9]. Neither different indexes presented in literature have been compared. Due to this lack of a universally accepted uniformity index, several approaches from the literature will be evaluated in this article for their applicability for FIM nonwovens. The goal of this work is to find a method that is suitable for the evaluation of nonwovens with different mass weights, thicknesses, sizes, and fiber structures.

In this article, first the current state of the art will be analyzed, followed by a preselection of the methods. Then the preselected methods will be analyzed with simulated images of different uniformities and images of several real nonwovens. Finally, the results will be presented and the applicability for the use case of evaluating FIM nonwovens will be discussed. 


\section{Evaluation of the state of the art}

\subsection{Analysis}

There is a lot of literature on the analysis of basis weight uniformity evaluation based on different approaches. Quite often, basis weight nonuniformity or irregularity of a nonwoven fabric is measured by physical methods, e.g., the cut-andweight method [10] which is probably the most used. For this method, samples are selected from different parts of a nonwoven web and weighed. The index of uniformity is then calculated by using the coefficient of variance (CV) of the samples' weight. However, the calculated CV depends highly on the number and size of the taken samples. Another disadvantage of this physical approach is the destruction of the tested nonwoven, which makes the method not applicable for an online measurement of basis weight uniformity.

Therefore, as the development of machine vision evolved, more and more nondestructive optical-based methods to measure weight uniformity were developed. In these works, the nonwoven fabrics were uniformly illuminated by transmitted or reflected light. In areas with higher basis weight, less light can pass the fabric than in areas with lower basis weight which causes a difference in gray values of the image.

A lot of these optical methods also rely on the calculation of the CV of light intensity transmitted through the nonwovens $[11,12]$. Parikh et al. used transmitted light images from an 8-bit monochrome camera to represent the basic weight of nonwovens and to calculate the $\mathrm{CV}$ in gray levels of single pixels for each image [13]. It has been shown that the $C V$ value of small samples $(0-25 \mathrm{~mm})$ decreases with increasing sample size and a minimum sample size is required. However, the $\mathrm{CV}$ is a global measure of variability and it does not take into account the spatial distribution of the fiber mass in nonwovens (second-order effect).

For the investigated samples it was shown that the uniformity depends on the measured size scale, which means samples of a different size will yield a different uniformity index.

Rather than calculating the CV based on individual pixels, other approaches combine the calculation of $\mathrm{CV}$ with the quadrants methods. The quadrants method is often used in ecology to estimate spatial characteristics [14]. This method divides the image into different sub-windows of same size, which can be referred to as the digital equivalent of the physical cutand-weight method. Tascan et al. presented the quadrant CV method that uses the average gray value of each sub-window to represent its basic weight and calculate CV as "index" of uniformity [15]. Additionally, the index of dispersion (Id), which calculates variance instead of the standard variance, was used for the analysis of uniformity. A decrease in these two indexes resulted by a lower variance represents a more uniform sample. Emadi et al. also adopted Id as index for weight uniformity measurement [16]. The difference between Emadi et al. and Tascan et al. is mainly found in image preprocessing methods. Tascan et al. adopted the light equalized gray value images as the input image for analysis while Emadi et al. used a binary image obtained with a K-nearest-neighbors (KNN) binary segmentation algorithm.

To overcome the issue with spatial resolution of CV-based methods, new works determined the spatial resolution of images with the Poisson tests. In the study by Pourdeyhimi and Kohel, images are thresholded using a fixed threshold and divided into a different number $n$ of sub-windows and the area fraction in each is calculated [17]. Then the index of dispersion and the chi-square value to test for randomness for every $n$ are computed for the final calculation of the uniformity index. The final calculated index is normalized between 0 (clustered) and 100 (uniform). Amirnasr et al. extended this approach by using gray value images instead of binary images [9]. This reduces the possible loss of information due to the binarization process. Analog to the work of Pourdeyhimi, the final calculated uniformity index is also normalized from 0 (clustered) to 100 (uniform). Chhabra developed a method similar to the two aforementioned which relies on the surface relief area instead of the gray value to represent the uniformity of mass distribution [18]. At first, the surface relief of each quadrat is calculated. Then, the dispersion of the quadrat surface relief area is represented by the overall index of dispersion Id, which is calculated by pooling row-wise and column-wise variance and dividing it by mean quadrat relief of the image. Later, the results were normalized between -1 (uniform) and 1 (clustered) and the index is calculated for aggregated, random, and uniform distributions.

There are also many other researchers trying to measure the weight uniformity of fibrous materials by new approaches. Bouydain et al. analyzed the formation of paper, which is also depended on the uniformity of fiber distribution in the frequency domain by using the 2D discrete Fourier transform [19]. The method used in his work is based on computing and extracting the information on the radial profile of the power spectrum of images and evaluating parameters $K, a$, and $p 0$ obtained by curve fitting of the radial profile of the spectra. To calculate the radial profile, the $2 \mathrm{D}$ power spectrum of transmitted light images was calculated first. Then, the $2 \mathrm{D}$ power spectrum was converted to the $1 \mathrm{D}$ power spectrum by integrating the $2 \mathrm{D}$ power spectrum in different directions of all radii. The bigger the parameters $a$ and $p 0$, the better the formation and uniformity of the paper.

Thorr et al. calculated the co-occurrence matrix of transmitted light images in several directions to derive structural and mechanical parameters of the nonwovens [20]. Different parameters of the co-occurrence matrix can be calculated which characterize different properties of the structure. The energy is a measure for the homogeneity and can be used as a uniformity index. The higher the energy, the more homogeneous the texture.

Yang and Takatera used fractal dimensions to analyze the fractal characteristics of the contact surface of needle-punched nonwovens [21]. In this article, the fractal dimensions are calculated by the box-counting method and based on the type of analyzed images (binary image or gray value image), two different indexes $D_{a}$ (the fractal dimension of fiber area 
distribution) and $D_{g}$ (the fractal dimension of gray-scale distribution) are calculated.

Table 1 gives an overview of the different methods, the used preprocessing methods, and the materials used for evaluation.

\subsection{Preselection of methods}

To find a suitable method for the evaluation of fiber injection molded nonwovens, a screening of the different classes of methods presented earlier is conducted. For this screening, the criteria maturity, accuracy, robustness, and efficiency are evaluated. The maturity refers to the amount of usages of a method in the literature. Accuracy means the quality of evaluation results mentioned in the respective paper, whereas with the robustness the suitability of a method for the measurement of nonwovens with different sizes, mass weights, and fiber structures is evaluated. With efficiency, the needed computation resources for preprocessing and calculation of the index are rated. The result of this preselection is shown in Table 2.

As mentioned earlier, an important requirement for the method is the ability to evaluate the weight uniformity of different kinds of nonwovens regardless of their thickness and basis weight. The method based on fractal dimension relied on the contact characteristics between pressed materials and the screen of the measurement equipment. Hence, the thickness of the material will greatly influence the calculated results which will not be considered in this article. Similarly, the index presented by Bouydain et al. [19] is sensitive to the local variation of the analyzed images and the result is also sensitive to the preset parameters. However, there are no rules to determine them. In addition, in this work the method has only been evaluated for

Table 1. Overview of methods for the calculation of mass weight uniformity

\begin{tabular}{|c|c|c|c|}
\hline Method class & Method & Preprocessing & Investigated material \\
\hline $\begin{array}{l}\text { Coefficient of } \\
\text { variance }(\mathrm{CV})\end{array}$ & CV & - & $\begin{array}{l}\text { Lightly needled hydro-entangled cotton, cotton } \\
\text { web blends }\end{array}$ \\
\hline \multirow{6}{*}{$\begin{array}{l}\text { Quadrant-based } \\
\text { methods }\end{array}$} & Quadrants CV & Gaussian filter & Polypropylene nonwoven fabrics \\
\hline & $\begin{array}{l}\text { Quadrants Id (gray } \\
\text { value) }\end{array}$ & Gaussian filter & Polypropylene nonwoven fabrics \\
\hline & Quadrants Id (binary) & K-means clustering & Polypropylene spun-bonded nonwoven fabrics \\
\hline & Binary chi-square & $\begin{array}{l}\text { Histogram equalization, } \\
\text { binarization }\end{array}$ & Wet-laid nonwovens \\
\hline & Gray value chi-square & Histogram equalization & Wet-laid nonwovens \\
\hline & Surface relief chi-square & Histogram equalization & $\begin{array}{l}\text { Spun-bonded nonwovens, carded thermo-bonded } \\
\text { nonwovens, spun-laced nonwovens }\end{array}$ \\
\hline Fourier transform & $\begin{array}{l}\text { Slope of spectrum } \\
\text { function }\end{array}$ & Histogram equalization & Paper \\
\hline $\begin{array}{l}\text { Co-occurrence } \\
\text { matrix }\end{array}$ & Directional energy & - & $\begin{array}{c}\text { Heat-bonded nonwoven, needle-punched } \\
\text { nonwovens }\end{array}$ \\
\hline \multirow{2}{*}{$\begin{array}{l}\text { Fractal } \\
\text { dimension }\end{array}$} & $\begin{array}{l}\text { Fractal dimension of } \\
\text { area distribution }\end{array}$ & - & Needle-punched nonwoven felts \\
\hline & $\begin{array}{l}\text { Fractal dimension of } \\
\text { gray-scale distribution }\end{array}$ & - & Needle-punched nonwoven felts \\
\hline
\end{tabular}

Table 2. Evaluation of methods for the calculation of mass weight uniformity

\begin{tabular}{|c|c|c|c|}
\hline Method class & Maturity & Accuracy & Robustness \\
\hline Coefficient of variation (CV) & ++ & + & 0 \\
\hline Quadrant methods & ++ & 0 & + \\
\hline Fourier power spectrum & - & 0 & - \\
\hline Co-occurrence matrix & 0 & 0 & + \\
\hline Fractal dimension & 0 & 0 & + \\
\hline
\end{tabular}


paper. So, it will also not be analyzed in our work. The method based on the co-occurrence matrix can measure uniformity in different directions, but currently there is no formula that can integrate these directional indexes into an index to represent mass uniformity of the overall image. Therefore, this method will also not be considered. In pretrials for this work, it has also been found out that the KNN algorithm for the binarization of our images cannot converge in an acceptable time, so the result of the segmentation is not good which will mislead the analysis method. Therefore, the method described by Emadi et al. [16] will also not be considered further in this article.

Both remaining method classes are based on the quadrant method and CV (Id, respectively). The theory of the quadrants methods is concise and the relationship between calculated index and weight distribution is clearly comprehensible which helps with the analysis of the results. At the same time, the quadrants methods have been attempted on many different types of nonwoven fabrics with different web structures and fiber forms. After several tests in related papers, these methods can be considered very mature. Other than that, these methods are computationally efficient as the calculation is on the scale of quadrants. Based on this preselection, the following methods show the best potential to meet the specified requirements and will be analyzed more in detail in the following sections:

- Quadrants CV (based on Tascan and Nohut [15])

- Quadrants Id (based on Tascan and Nohut [15])

- Binary chi-square (based on Pourdeyhimi and Kohel [17])

- Gray value chi-square (based on Amirnasr et al. [9])

- Surface relief chi-square (based on Chhabra [18]).

\section{Experimental}

In this part, the analysis of the methods mentioned earlier will be presented. Simulated images are used to determine the behavior of the methods when evaluating images with different degrees of clustering. Additionally, three sets of real images will be evaluated with the methods to judge the robustness of the methods to samples of similar properties and whether they are capable in distinguishing varying fiber structures and their corresponding uniformities.

\subsection{Analysis of simulated images}

For the generation of simulated images in this article, an approach presented by Amirnasr et al. [9] is used. In this method, the number of pixel clusters and the cluster width factor (CWF), which controls the dispersion of pixels in each cluster, are two major parameters. Each cluster contains a predetermined number of white pixels and the radial distribution of distances between objects from the center of the cluster, which controls the dispersion of white pixels, follows a normal distribution function as follows:

$$
f(x)=\frac{1}{\sigma \sqrt{2 \pi}} e^{\frac{-(x-\mu)^{2}}{2 \sigma^{2}}}
$$

where $\mathrm{x}$ is the distance from center, $\mu$ is the average distance, and $\sigma$ is the standard deviation.

As the standard deviation increases, the dispersion of the cluster becomes more uniform. The standard deviation is controlled by the size of clusters and defined by the CWF:

$$
C W F=\frac{\sigma}{W}
$$

where $W$ is the area where one cluster occupied.

Maintaining a constant area fraction ( $20 \%$ white pixels), the spatial uniformity of images will increase if the cluster number $n$ or CWF is increased. By using these methods, a series of simulated images with different human-controlled spatial characteristics can be quickly generated. The image size of the generated images is $600 \times 600$ pixels. Besides the uniformity of pixels in each cluster, the location of the center point of each cluster can also be controlled. Two subsets of images can be created: a random set where center points are selected randomly as shown in Figure 1 and a uniform set where cluster center points are uniformly distributed as shown in Figure 2. In each set, five different cluster numbers (1, 9, 100,900 , and 10,000) and nine different CWFs $(0.1,0.5,1,2$, $3,4,5,10$, and 50 ) were used. For the random set of images, five different images for every parameter set $n$ and $C W F$ were generated. Hence, the obvious change of image uniformity in each subset of images, the image sets can be used to evaluate the accuracy of different analysis methods. Additionally, due to the different distribution of clusters between uniform set and random set, especially the different distribution of clusters on the five different simulated images with the same parameters in the random set, the image sets can be used to evaluate the robustness of different methods to the change of structure of nonwovens.

\subsection{Analysis of real images}

As mentioned earlier, three sets of real nonwovens will be used to analyze the uniformity of evaluation methods. These three sets consist of different types of nonwovens (cf. Figure 3). The first set A (images A1-A5) consists of five samples of fiber injection molded nonwovens with a basis weight of around 1.250 $\mathrm{g} / \mathrm{m}^{2}$ made of a 2.400 tex glass fiber produced on a FIM test rig at wbk Institute of Production Science. The second set $B$ (images B1-B5) contains five samples of a needled glass fiber mat with $610 \mathrm{~g} / \mathrm{m}^{2}$ basis weight including a second layer of a coarse weave produced by P-D Glasseiden $\mathrm{GmbH}$ (Oschatz, Germany). Set C (images C1-C5) contains five powder mats with a basis weight of $450 \mathrm{~g} / \mathrm{cm}^{2}$ with very good surface distribution also produced by P-D Glasseiden $\mathrm{GmbH}$. The nonwoven samples in all sets have a size of $400 \times 400 \mathrm{~mm}$. The uniformity of the nonwovens of sets $B$ and $C$ is obviously higher than for set $A$ as they are produced with an industrial scale continuous web forming machine. In addition, the fiber distribution uniformity of different images within sets $B$ and $C$ is not very different. Therefore, the images within the same set can be used to evaluate the reliability of different methods and the images from different sets can be used to evaluate the accuracy of different methods (Figure 3 ). 
Cluster number $n$
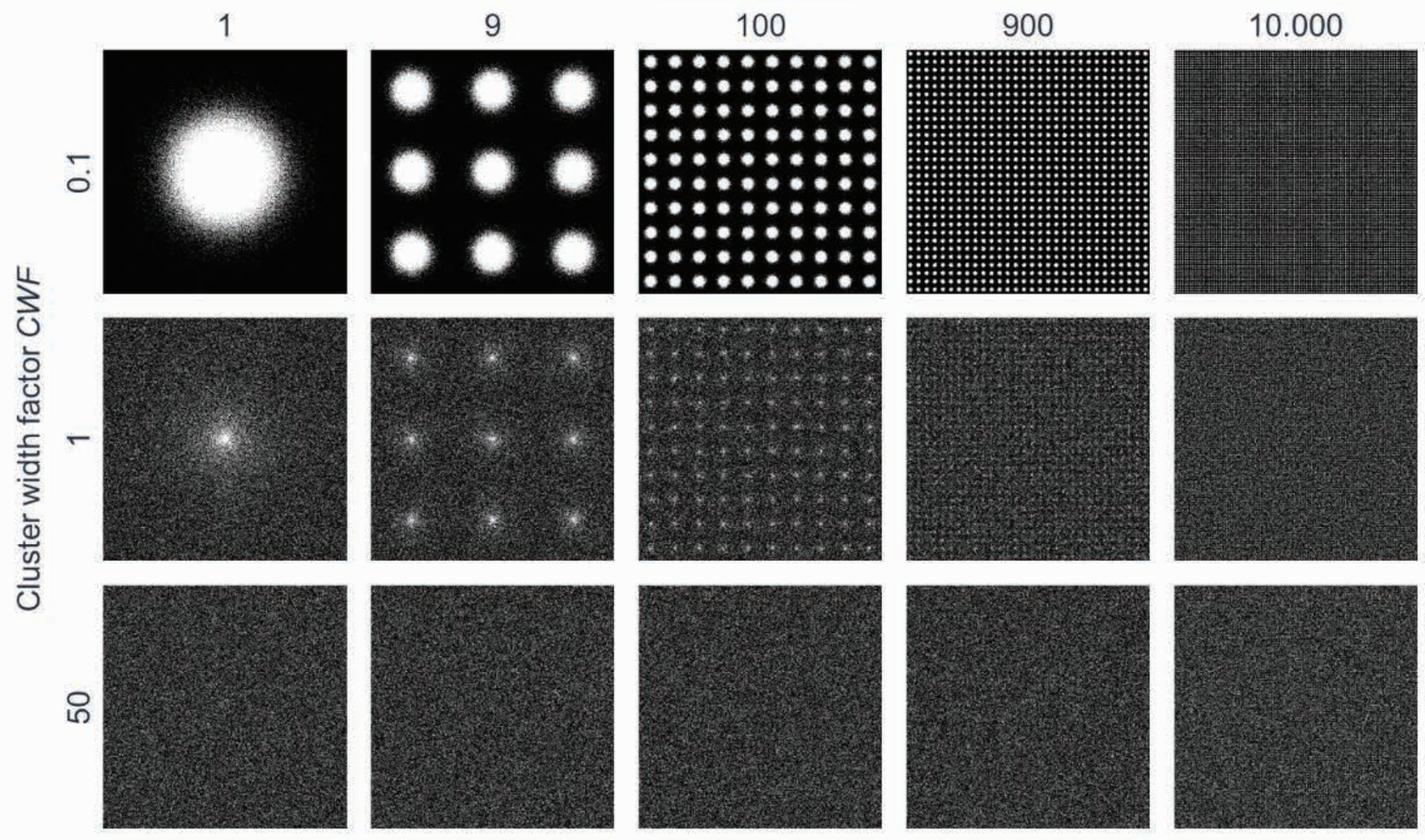

Figure 1. Simulated images: uniformly arranged clusters.
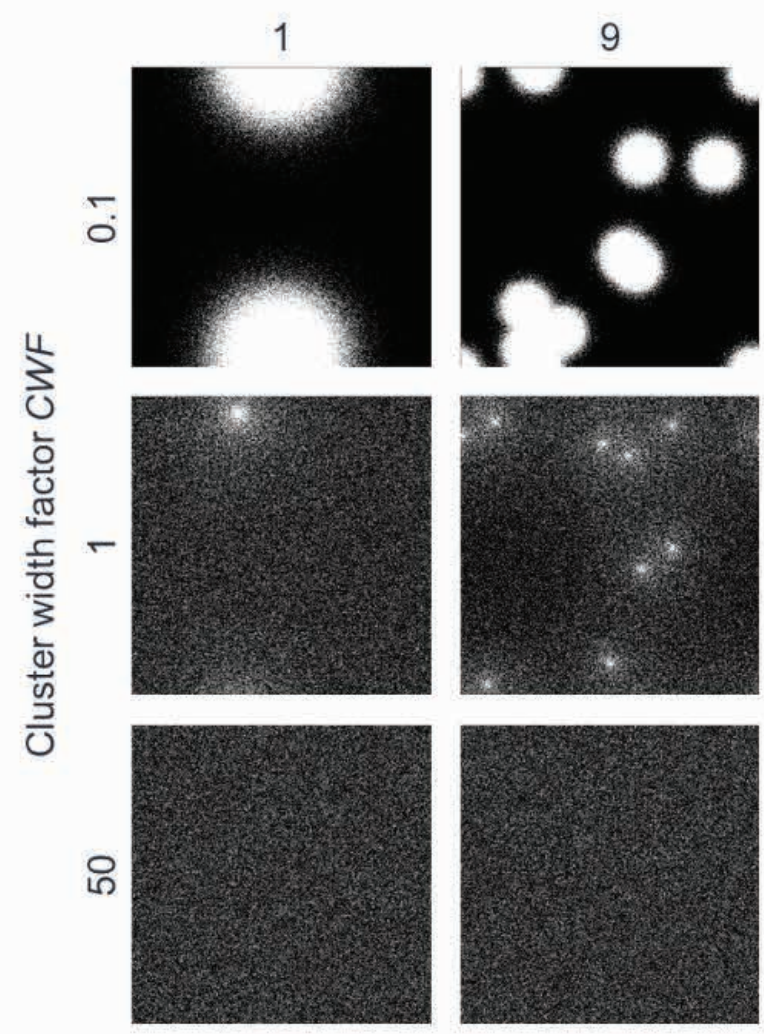

Cluster number $n$
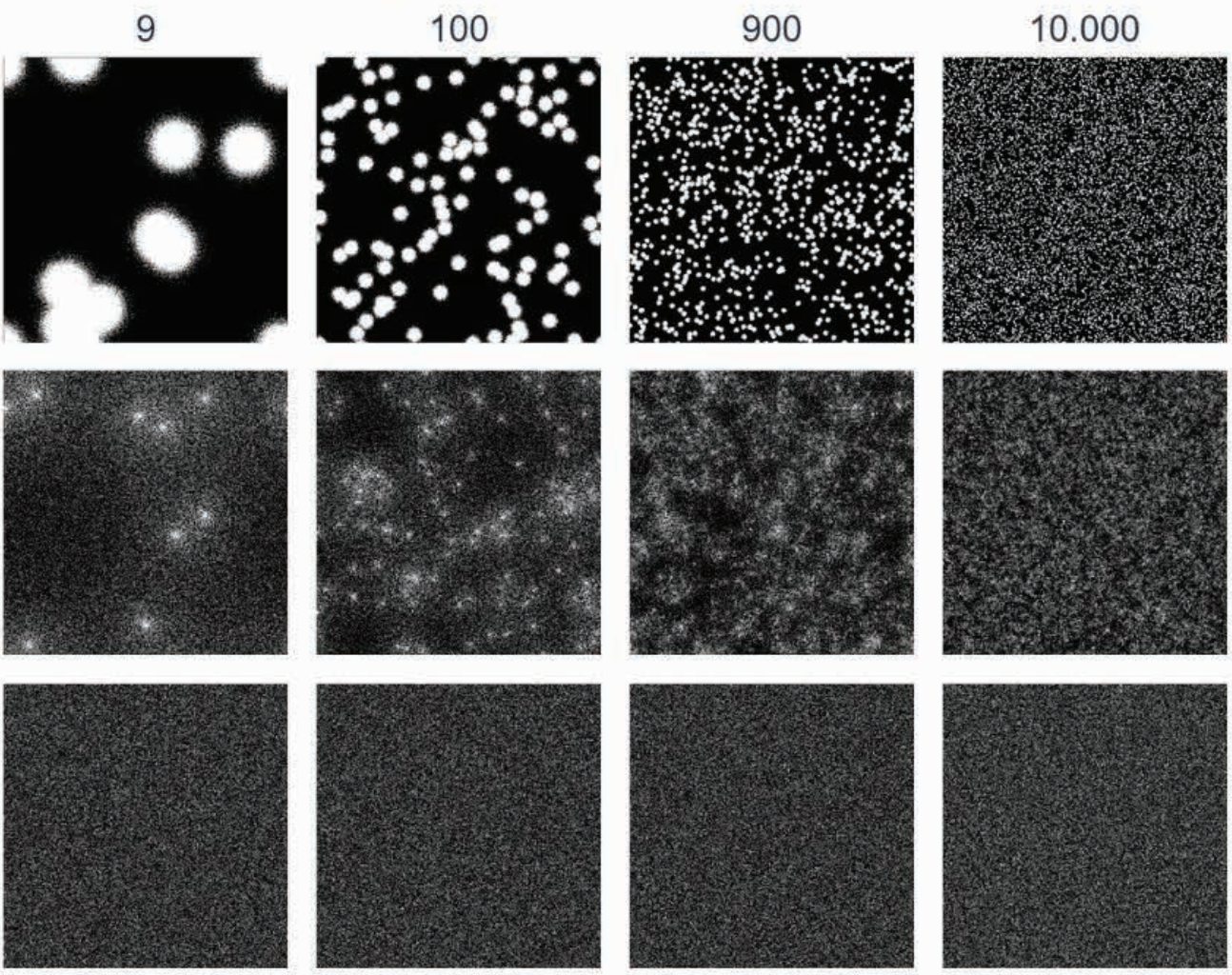

Figure 2. Simulated images: randomly arranged clusters. 
The images of the real nonwovens are acquired with the mold monitoring system described in the study by Moll et al. [8]. It consists of a CMOS camera with a resolution of $9 \mathrm{MP}$ and an LED backlighting to acquire transmitted light images. The acquired images were cropped to the size of the samples resulting in an image size of $2,000 \times 2,000$ pixels.

Before the application of the evaluation methods on the real images, they should be preprocessed to remove possible artifacts during image acquisition such as noise and uneven lighting. As discussed earlier, in the related works, the preprocessing methods can be summarized in three main steps. First, the acquired images will be filtered by a Gaussian filter with a kernel size of $3 \times 3$ pixels to remove noise. Second, histogram equalization will be adopted to remove any deviations caused by the illumination system. Third, for the methods using binary images as input, the binarization is executed similar to that described in the corresponding papers. However, despite histogram equalization, it is crucial to choose image acquisition parameters, especially exposure time, in a suitable range to prevent a loss of weight information by overexposed areas.

\section{Results and discussion}

\subsection{Analysis of simulated images}

The sets of simulated nonwoven images with distinguishable degrees of uniformity were evaluated by original $\mathrm{CV}$ and the five preselected indexes. The evaluation results of the simulated images having 100 clusters are presented in Figure 4. The five images in the random image set with the same properties were evaluated individually and the calculated mean and standard deviation are shown in Figure 4. Original CV will be used as reference for the other five different methods. The CV does not change as the CWF and therefore uniformity increases. As the calculation of $C V$ relies on the pixel-wise gray value change, it changes only with varying area fraction in the case of images with only black and white pixels as is the case for the simulated images. As the area fraction of the simulated images is constant by definition (see earlier), the CV value remains constant.
The calculation of the other indexes relies on the quadrants method, separating the image in $8 \times 8,64$ sub-windows in the case of this investigation. Similarly mentioned in the review of the state-of-the-art $\mathrm{CV}$ and Id trend toward 0 with higher uniformity, whereas the binary and gray value chi-square indexes are normalized between 0 and 100 with values closer to 100 , meaning better uniformity. The surface relief chi-square index reaches the best uniformity at -1 . Figure 4 shows all indexes trending toward higher uniformity with increasing CWF as it could have been expected. A uniform distribution of clusters leads to a higher uniformity than a random distribution with the same cluster number and CWF. Quadrants Id and binary chi-square index reach values close to their maximum value for small CWFs. This mean that the indexes have a very little resolution for evaluating images with a high uniformity as the change in the indexes value is very little for a varying degree of uniformity. From the results of the evaluation of the random images (cf. Figure 4), it is also obvious that the standard deviation of the uniformity index calculated from five simulated images with same parameters is very high for the surface relief chi-square index and the gray value chi-square index. Especially for results in the middle of the indexes range, the standard deviation is of drastically high magnitude. In addition, for the surface relief chi-square index the result for random images with a CWF smaller than 1 is constantly 0.5 , which means that the images are considered clumped (cf. [18]) and no further measure of uniformity can be attributed. The quadrants $\mathrm{CV}$ index shows a constant behavior for increasing CWF and a standard deviation less than $5 \%$. Therefore, the quadrants $\mathrm{CV}$ index is suitable for the evaluation of the whole range of simulated images.

\subsection{Analysis of real images}

Additionally, the three sets of real nonwoven images with distinguishable degrees of uniformity, different thicknesses, and different mass weights were evaluated using the same six methods. The images were also subdivided into 64 subwindows as was the case for the simulated images. Figure 5 shows the mean index value and standard deviation calculated from the results of the five images in each set. All the indexes show a lower uniformity for set $A$ than for sets $B$
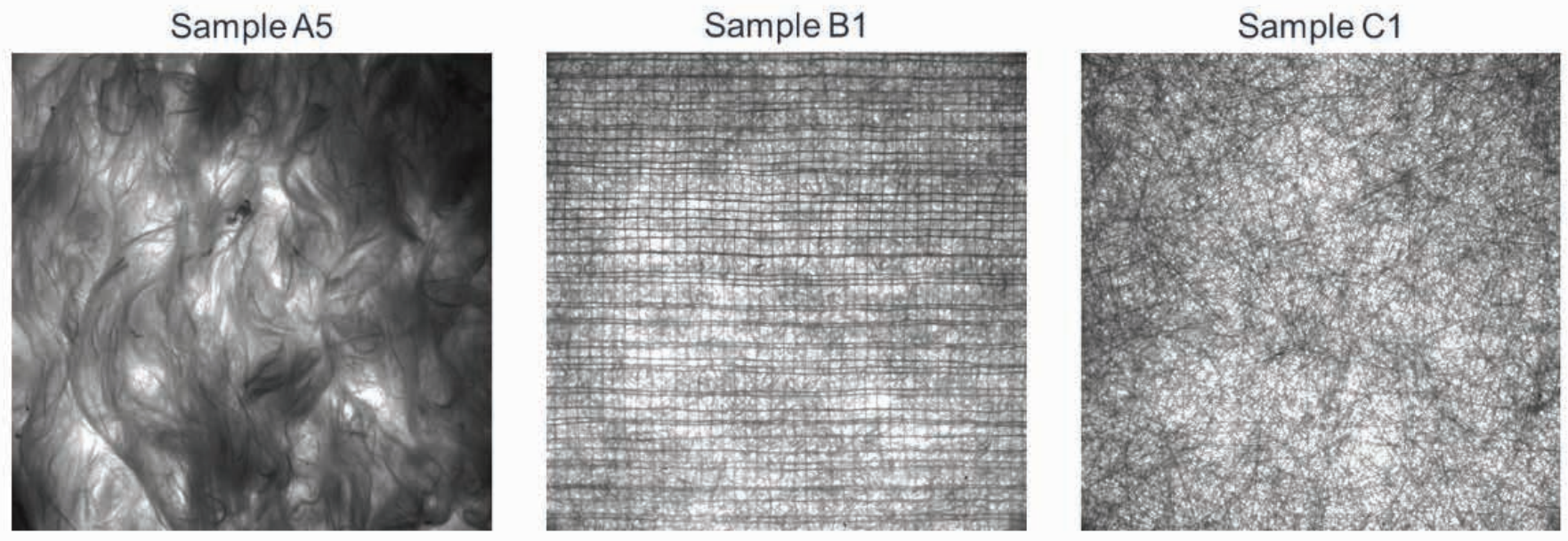

Figure 3. Real nonwoven images: samples from sets A, B, and C. 
and $\mathrm{C}$, which corresponds to the subjective impression looking at the transmitted light images (see Figure 3). Set $C$ is rated slightly better than set $B$ caused by the weave structure of the nonwovens in set $B$. In the case of all indexes, this relative ranking remains unchanged. Also, all indexes show a higher standard variation for set $A$ which can also be explained by the discrete manufacturing process of these nonwovens leading
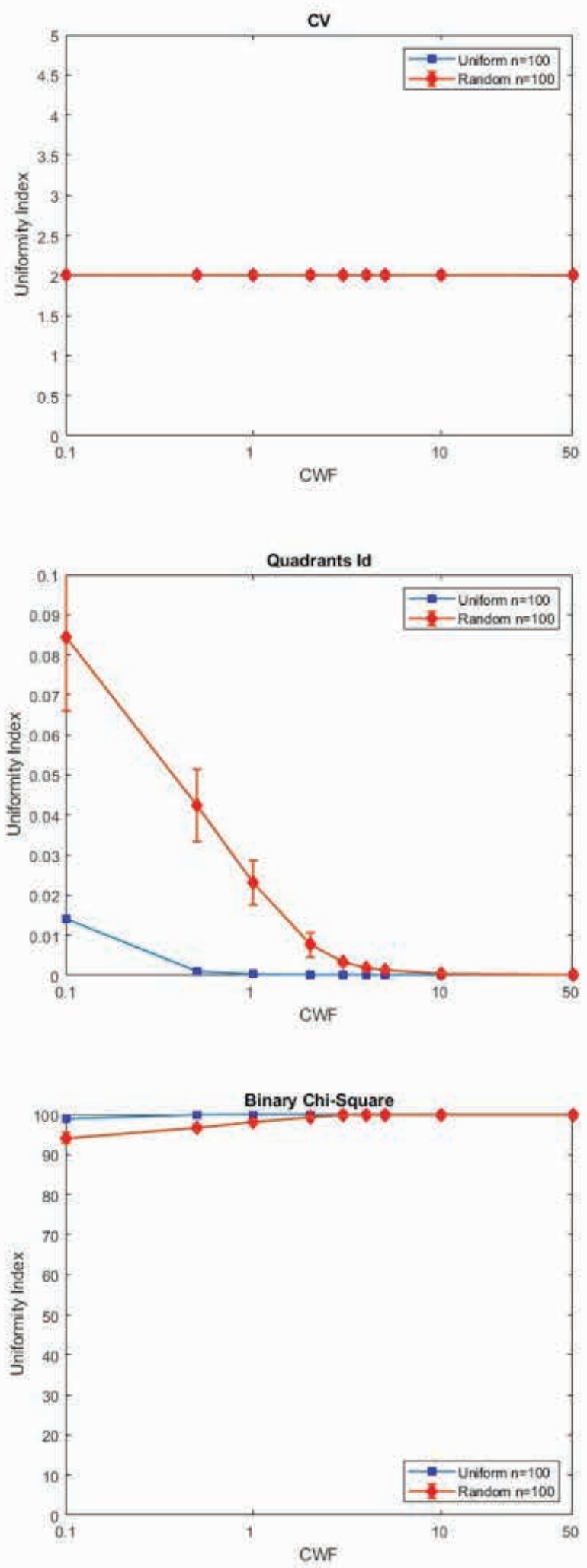

Figure 4. Evaluation results of simulated images with 100 clusters. to less uniform fiber structure than continuously produced nonwovens. As for the simulated images the binary chi-square index shows the highest score on its scale, with the values for sets $B$ and $C$ close to the maximum value of 100 and difficult to distinguish. The surface relief chi-square index and the gray value chi-square index show a good sensitivity to the uniformity changes of the different sets but the standard deviation for set
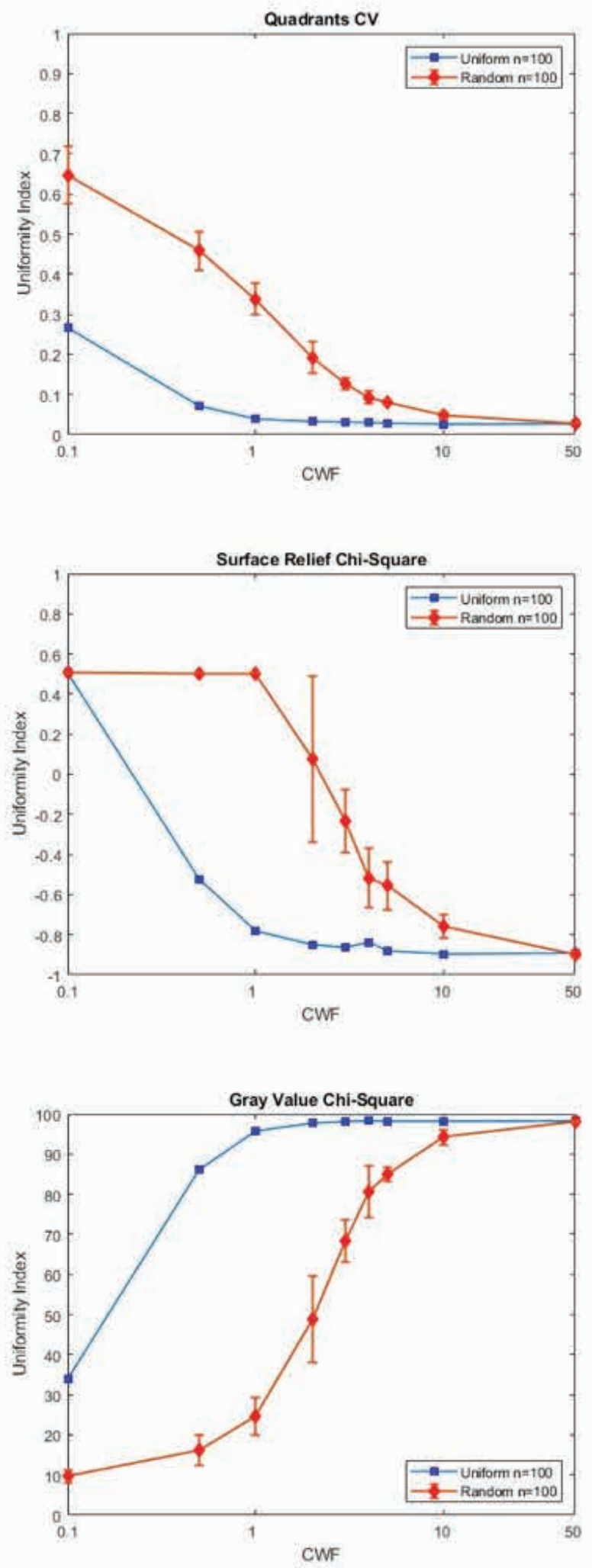

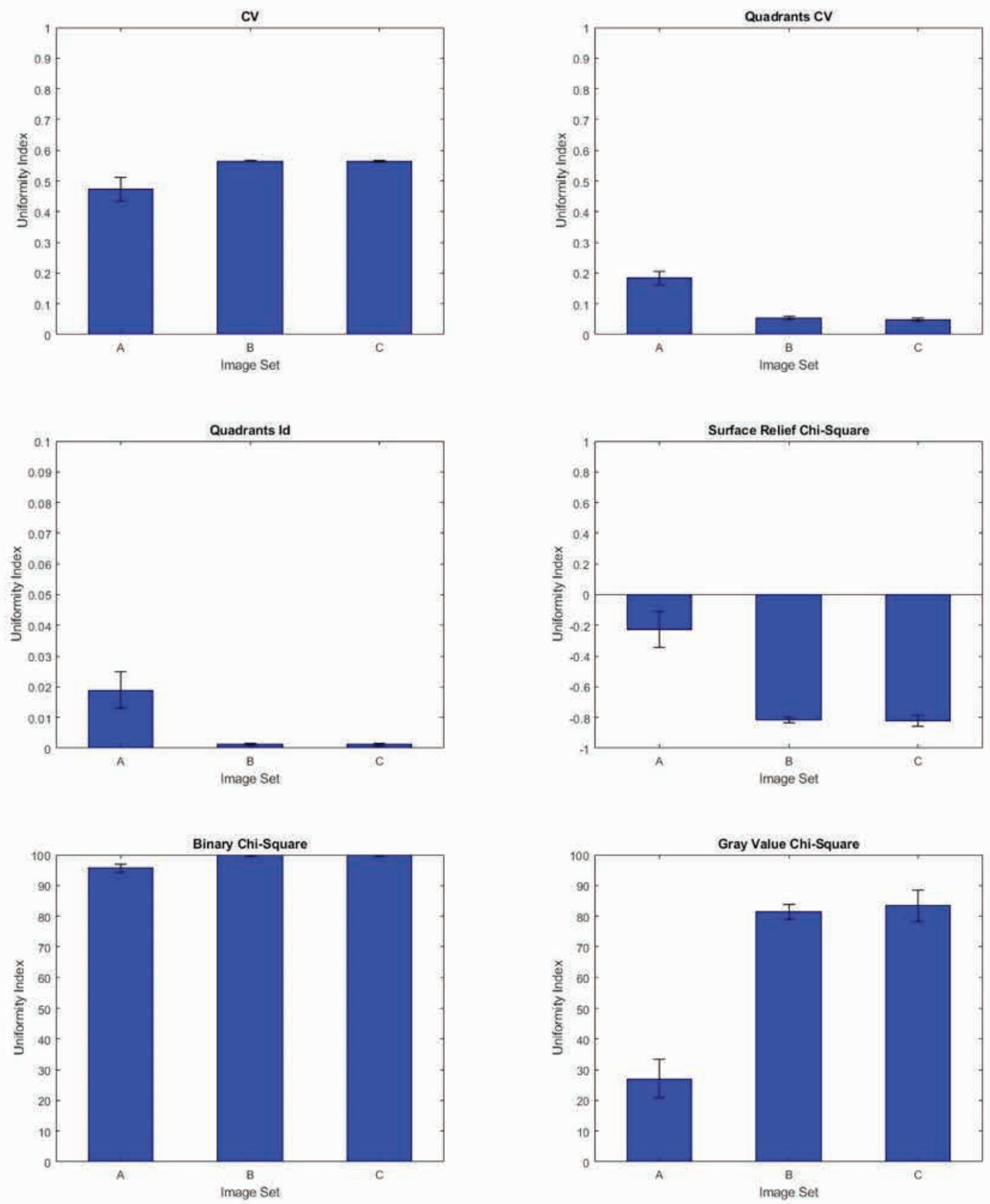

Figure 5. Evaluation results of real nonwoven images.

B and C is higher than $5 \%$. Quadrants CV and quadrants Id also show a good applicability for the distinction of different image sets.

Figure 6 shows the uniformity indexes and computational time for calculating the uniformity index as a function of quadrant number on the real nonwoven image B2. The method $\mathrm{CV}$ is used as a reference. The $1,500 \times 1,500$ pixels real images are evenly divided by $4-900$ quadrants. As the quadrants number increases, the size of the quadrants decreases from $750 \times 750$ to $50 \times 50$ pixels. As shown in the figure, with increasing quadrant number and therefore decreasing quadrant size the resulting uniformity indexes of all five quadrant methods tend to become more nonuniform. This can be explained by the fact that the calculation of the mean in larger quadrants will intuitively compensate the variety of gray value between 
smaller quadrants, which causes a smaller standard variance of gray value between quadrants. The sensitivity to the quadrants number and quadrants size is the major problem of quadrants methods. This leads to the constraint that quadrant number should be kept at a constant value when using the uniformity indexes to compare nonwovens. Based on the results of our evaluation on simulated and real images with a constant quadrants number (here, $8 \times 8$ sub-windows), the methods
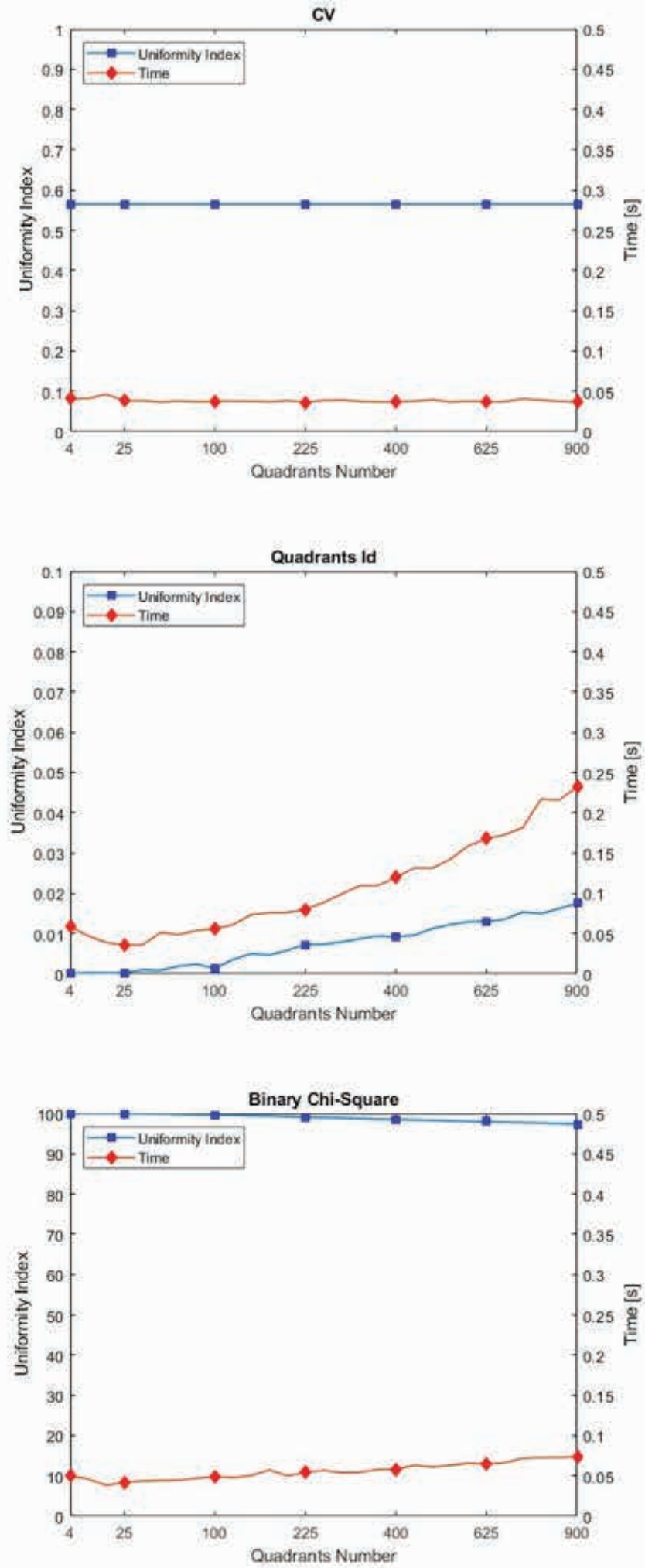

Figure 6. Evaluation of quadrants number on indexes. can accurately and robustly evaluate the weight uniformity of different nonwoven fabrics. Hence, for the measurement the nonwovens sample with same size can be divided into constant quadrants number to prevent that the variety of quadrants number influences the measurement results.

In real application, the sample size can differ. If a constant quadrants number is used, as the sample size increases, the
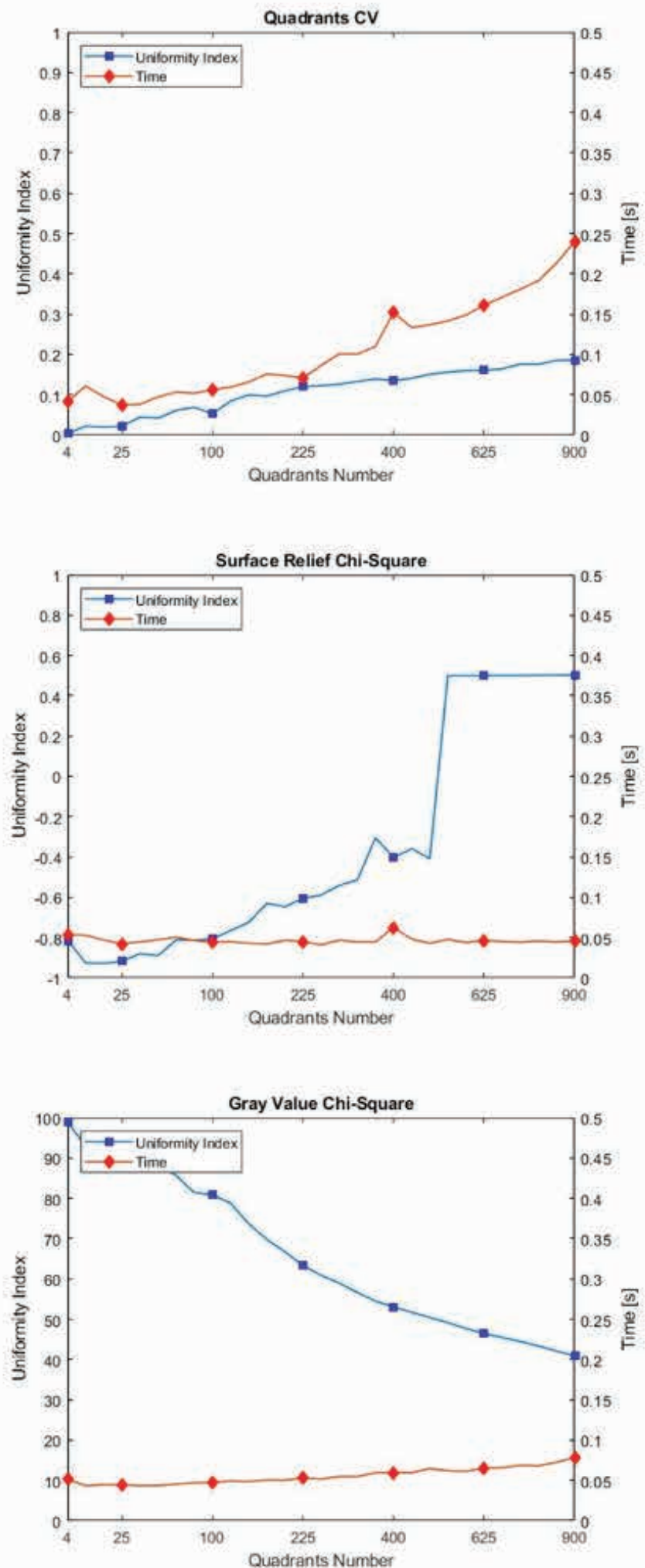
quadrants size will also increase. To investigate this effect, different sample sizes have been analyzed with a constant quadrants number of 64 . Figure 7 shows the calculated indexes based on the samples with a size increase from $500 \times 500$ to $1,500 \times 1,500$ pixels. The results of different methods show no significant change with increasing sample size. Therefore, it can be stated that for sample size bigger than $500 \times 500 \mathrm{~mm}$, the indexes are size invariant.
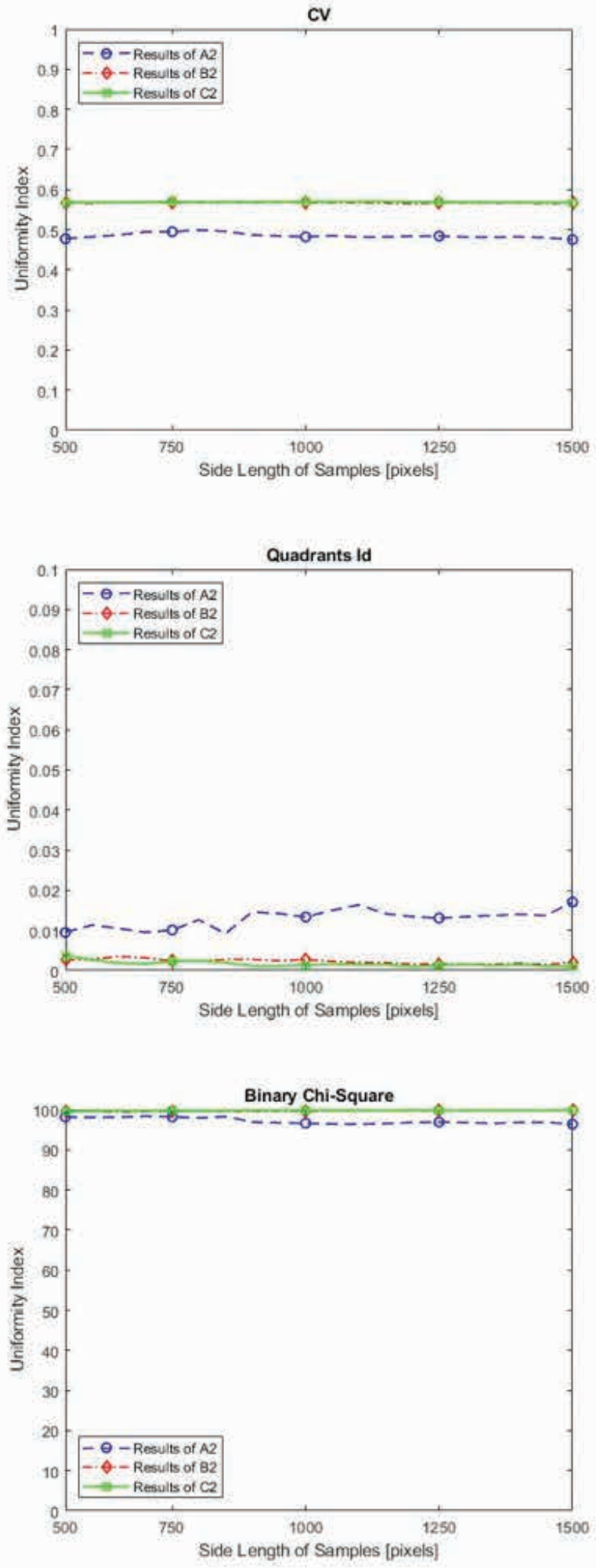

Figure 7. Evaluation of sample size effect on indexes.

\section{Conclusions}

In this study, different quadrant-based methods have been implemented and compared based on the analysis of simulated and real nonwoven images. It has been shown that with a sample size big enough to represent the uniformity of nonwoven fabrics, the quadrants methods can determine the weight distribution uniformity reliably with constant quadrants
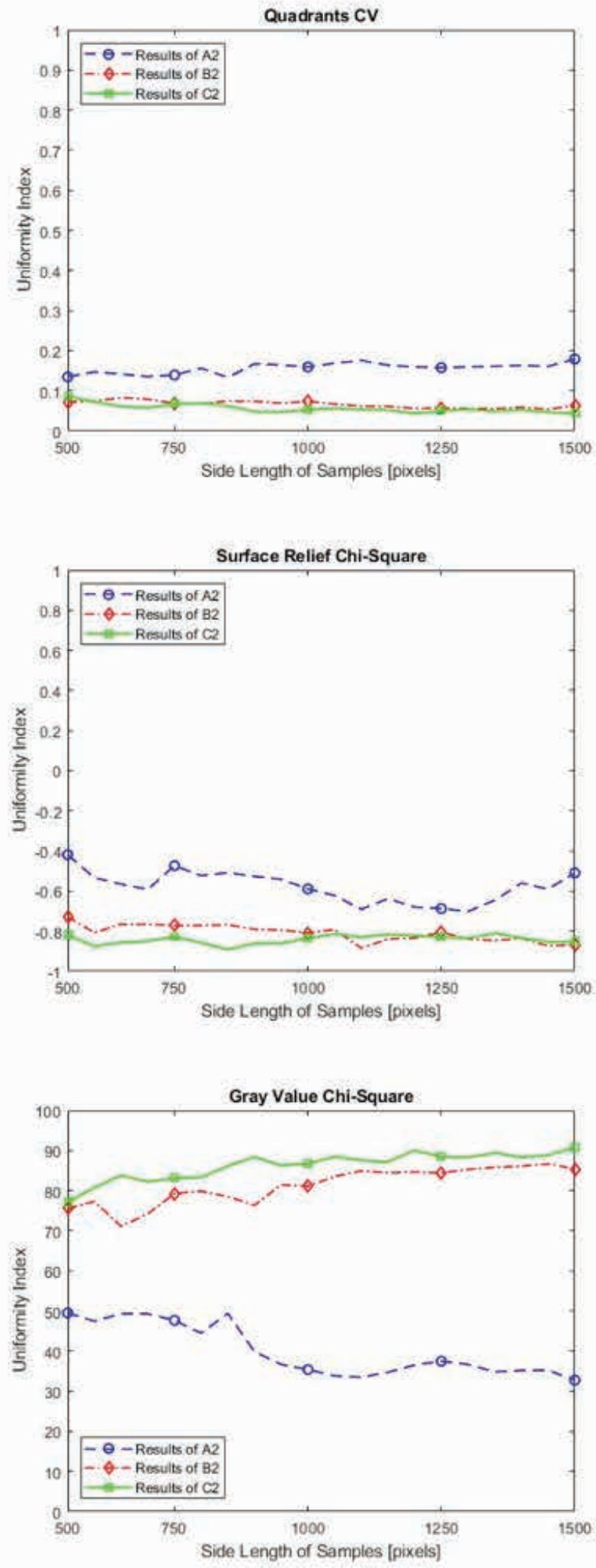
number. The computing time for all methods is very reasonable. Considering the requirement to evaluate nonwovens of different thicknesses and mass weights, it has been found that the quadrants Id and binary chi-square method have a low measurement resolution for the analyzed images, making it difficult to analyze different nonwovens. The surface relief chi-square and gray value chi-square method show a lack of robustness in the medium range of the indexes. The quadrants CV method performs best regarding the set requirements of analyzing nonwovens with different mass weights, thicknesses, sizes, and fiber structures. It shows a good measurement resolution for the whole range of investigated images and a good robustness. Additionally, the index is not significantly influenced by the sample size. However, it is crucial to select correct image acquisition parameters for the respective nonwoven.

The findings of our study indicate that quadrant-based methods can be used for online monitoring of nonwoven basis weight uniformity. Based on the results, the quadrants CV method will be used for further analysis of fiber injection molded nonwovens. It is planned to use this index to investigate the influence of process parameters on the uniformity of FIM nonwovens. The findings of this research can be used for quality inspection of nonwoven sheets, e.g., for composite applications. Additionally, the approach can be adapted for online inspection systems in nonwoven production machinery.

\section{Acknowledgments}

This publication was partly written in the framework of the Profilregion Mobilitätssysteme Karlsruhe which is funded by the Ministry of Science, Research and the Arts in Baden-Württemberg. The other part was supported by the German Research Foundation (DFG: Deutsche Forschungsgemeinschaft; project no. 439709829).

\section{References}

[1] Fleischer, J., Teti, R., Lanza, G., Mativenga, P., Möhring, H.C., et al. (2018). Composite materials parts manufacturing. CIRP Annals 67(2), 603-626.

[2] Cherif, C. (2011). Textile Werkstoffe für den Leichtbau: Faserstoffe, Halbzeuge und Preforms: Technologie und Eigenschaften. Springer (Berlin).

[3] Stegschuster, G., Schlichter, S. (2018). Perspectives of web based composites from RCF material. IOP Conference Series: Materials Science and Engineering 406, pp. 1-6.

[4] Cherif, C. (Ed.). (2013). Leichtbau mit Textilverstärkung für Serienanwendungen: Bindematerialien - Textile Preforms - Verbundbauteile; Buch zum DFG-AiF-Clustervorhaben Leichtbau und Textilien. Verl. Wissenschaftliche Skripten.

[5] Förster, E. DE 10324735 B3 (Germany). May 30, 2003.

[6] Fleischer, J., Förster, F., Dackweiler, M. (2015). Materialeffiziente hybride Preforms aus Lang- und Endlosfasern. Lightweight Design, 2015(6), 14-19.
[7] Dackweiler, M., Fleischer, J. (2017). Automated local reinforcing of glass fiber-injection-molded preforms with carbon fiber tapes. Proceedings of the 15th Japan International SAMPE Symposium \& Exhibition.

[8] Moll, P., Schäfer, A., Coutandin, S., Fleischer, J. (2019). Method for the investigation of mold filling in the fiber injection molding process based on image processing. Procedia CIRP 86, 156-161.

[9] Amirnasr, E., Shim, E., Yeom, B.-Y., Pourdeyhimi, B. (2014). Basis weight uniformity analysis in nonwovens. The Journal of the Textile Institute, 105(4), 444-453.

[10] Ericson, C. W., Baxter, J. F. (1973). Spunbonded nonwoven fabric studies: characterization of filament arrangement in the web. Textile Research Journal, 43, 371-378.

[11] Boeckerman, P. A. (1992). Meeting the special requirements for on-line basis-weight measurement of lightweight nonwoven fabrics. Tappi Journal, 75, 166-172.

[12] Veerabadran, R., Davis, H. A., Batra, S. K., Bullerwell, A. C. (1996). Devices for on-line assessment of nonwovens' basis weights and structures. Textile Research Journal, 66(4), 257-264.

[13] Parikh, D. V., Bresee, R. R., Sachinvala, N. D., Crook, L., Muenstermann, U., et al. (2006). Basis weight uniformity of lightly needled hydroentangled cotton and cotton blend webs. Journal of Engineered Fibers and Fabrics, 1(1), 4761.

[14] Greig-Smith, P. (1964). Quantitative plant ecology. University of California Press (Los Angeles).

[15] Tascan, M., Nohut, S. (2015). Nondestructive prediction of areal weight, grab tensile strength and elongation at break of polypropylene (PP) spunbond nonwoven fabrics using digital image analysis. Tekstil ve Konfeksiyon, 25(1), 2432.

[16] Emadi, M., Tavanaie, M. A., Payvandy, P. (2018). Measurement of the uniformity of thermally bonded points in polypropylene spunbonded non-wovens using image processing and its relationship with their tensile properties. Autex Research Journal 18(4), 405-418.

[17] Pourdeyhimi, B., Kohel, L. (2002). Area-based strategy for determining web uniformity. Textile Research Journal, 72(12), 1065-1072.

[18] Chhabra, R. (2003). Nonwoven uniformity - measurements using image analysis. International Nonwovens Journal, 12(1), 43-50.

[19] Bouydain, M., Colom, J. F., Navarro, R., Pladellorens, J. (2001). Determination of paper formation by Fourier analysis of light transmission images. Appita Journal, 54(2), 103-105+115.

[20] Thorr, F., Drean, J. Y., Adolphe, D. (1999). Image analysis tools to study nonwovens. Textile Research Journal 69(3), 162-168.

[21] Yang, X., Takatera, M. (2011). Fractal characteristics of contact surface of needle punched nonwovens. In: Song, L., Xiong H., (Eds.). Advances in computer science, environment, ecoinformatics, and education, Vol. 215, Springer (Berlin Heidelberg). 